anomalies due to the Christiansen effect are omitted. The final chapter outlines other absorptions, for example magnons, which may confuse interpretation.

Overall the book is a useful reference for the research worker active in the field, but its uncritical presentation of material and references is likely to persuade others, particularly undergraduates, that there are easier fields for study.

D. BLOOR

\section{Particle Collisions}

Dissociation in Heavy Particle Collisions. By G. W. McClure and J. M. Peek. Pp. xi +198. (Wiley: New York and London, June 1972.) £5.50.

Excitation in Heavy Particle Collisions. By E. W. Thomas. Pp. xiv +436. (Wiley: New York and London, August 1972.) $£ 9.45$.

THE explosive rate of growth of research activity in many fields during the past twenty years has raised increasingly acute problems of dissemination of information. Even when the results of the research are published in the standard journals the sheer volume of publication makes it increasingly difficult to extract information in a specific field. Much of the work never gets published in this form. It remains in conference reports, preprints, or even in the notebooks of the research worker. More than twenty years ago J. D. Bernal remarked that it was becoming easier sometimes for oneself to repeat an experiment than to retrieve information about the results of earlier experiments from the literature, and since then the problem has become much more acute.

This situation has led to the growth of data compilations in various fields, continually up-dated as the results of more work becoming available, and the two books reviewed here are essentially such compilations in two rather narrow fields related to the collisions of atomic and molecular particles. Such collision processes are of great importance in many fields-gas discharge plasmas, nuclear fusion reactors, high voltage breakdown, high energy accelerators, aurorae, astrophysics, for example.

The two books have been produced as part of a project directed by the Atomic and Molecular Processes Information Centre at the Oak Ridge National Laboratory. The project itself forms part of a wider initiative of data collection being undertaken by US Government agencies associated with science and technology.

Similar compilations in the past have often been limited in their usefulness because they have been collected uncritically without much regard for the care with which the measurements have been carried out and accepting the assessment of errors given by the authors of the papers. Indeed, with the growing emphasis on conciseness, published accounts of experiments often omit the essential detailed information necessary for an independent assessment of the significance of a particular measurement. The objectives of the present project are defined as "the collection, evaluation and critical review of data in specialized topics of heavy particle atomic and molecular collisions", and the authors seem to have given considerable attention to the critical assessment of each measurement. Where points in published work are obscure, or vital information lacking, they have written round extensively to authors. Sets of criteria have been formulated for judging the reliability of data. One feels that measurements able to pass these stringent tests can be accepted with confidence and that both books do indeed fulfil the basic aim of making reliable data available in a form useful especially for scientists and engineers who wish to use them.

These books are, however, much more than data compilations. Both contain detailed critical assessments of experimental methods written for the benefit of the specialist planning further work, and should thus be of value both for the established research worker and for research students entering the field.

The book by Drs McClure and Peek provides indexes which show where specific data on dissociation in heavy particle collisions can be found but does not actually give any data. It includes, however, a qualitative discussion of the results of experiments on the cross sections for fragmentation processes of various kinds in such collisions and on the energy and angular distributions of the fragments. It reviews the results of theoretical calculations as well as experimental measurements.

The book by Dr Thomas on excitation processes in heavy particle collisions includes for each entry in its extensive index, an assessment of the extent to which the work fails to satisfy the specific criteria formulated for judging reliability. In addition, however, it includes many tables giving cross sections for the excitation of particular states and the polarization of the subsequent radiation for processes involving excitation of both the projectile and target particles, and for excitation of the projectile following charge exchange. The data are given as a function of the projectile energy for $\mathrm{H}, \mathrm{H}_{2}+, \mathrm{H}_{3}+, \mathrm{He}^{+}$and heavier projectiles on targets ranging from atomic hydrogen to metallic vapours and light hydrocarbons. Data of this type on approximately 450 excitation processes are included and the main features of each type of data are summarized critically.

In all, this represents a prodigious amount of effort and foreshadows types of presentation likely to become general for the collection of scientific information.

E. H. S. Burhop

\section{Photoelectron Spectroscopy}

Photoelectron Spectroscopy. By A. D. Baker and D. Betteridge. Pp. $x+180$. (Pergamon: Oxford and New York, August 1972.) $£ 3.50$.

Photoelectron spectroscopy is a technique for studying the binding energies of electrons in the various orbitals or shells of molecules by measuring the energies they acquire when ejected by a photon of known frequency $\left(E_{\mathrm{B}}=h \nu-\frac{1}{2} \mathrm{mv}^{2}\right)$. The subject is conveniently divided into ultraviolet and $X$-ray photoelectron spectroscopy, the former being concerned with the electrons in the orbitals of the valence shell and the latter with the core or inner shell electrons. The technique has proved to be far more generally applicable than conventional spectroscopic methods and in the past ten years has had remarkable success in revealing an enormous amount of detail about the electronic structure of matter.

The first book on this topic which was written by K. Siegbahn and collaborators dealt mainly with the use of soft $\mathrm{X}$-rays (ca. $1,000 \mathrm{eV}$ photons) to study core electron behaviour. The second book by D. W. Turner and others gave an account of the use of ultraviolet photons mainly the $58.4 \mathrm{~nm}$ helium line (21 eV photons) for examining the valence orbitals of molecules. The present book attempts to give an integrated account of both these aspects of the subject and to describe the main lines along which development has taken place in the last decade. It is thus a valuable introductory manual in the field, since apart from the 300 references to the more important papers on the subject it contains many tables of data which the authors have found to be of value in operating photoelectron spectrometers over a number of years. The theoretical and practical aspects of the subject are discussed with a view to showing how the results can be applied to the elucidation of organic structures, qualitative identification and quantitative analysis. The field holds great and growing interest for both chemists and physicists, and this condensed assessment of its present status is bound to be of value to them.

W. C. Price 